COMMUNICATIONS IN

ANALYSIS AND GEOMETRY

Volume 13, Number 5, 1057-1075, 2005

\title{
Rigidity of Differentiable Structure for New Class of Line Arrangements ${ }^{1}$
}

\author{
Shaobo Wang, Stephen S.-T. Yau ${ }^{2}$
}

\begin{abstract}
An arrangement of hyperplanes is a finite collection of $\mathbf{C}$-linear subspaces of codimension one in a complex vector space $\mathbf{C}^{l}$. For such an arrangement $\mathcal{A}$, there is a natural projective arrangement $\mathcal{A}^{*}$ of hyperplanes in $\mathbf{C P}^{l-1}$ associated to it. Let $M(\mathcal{A})=\mathbf{C}^{l}-$ $\bigcup_{H \in \mathcal{A}} H$ and $M\left(\mathcal{A}^{*}\right)=\mathbf{C} \mathbf{P}^{l-1}-\bigcup_{H^{*} \in \mathcal{A}^{*}} H^{*}$.

One of central topics in the theory of arrangements is to find connections between the topology or differentiable structure of $M(\mathcal{A})$ (or $M\left(\mathcal{A}^{*}\right)$ ) and the combinatorial geometry of $\mathcal{A}$. A partial solution to this problem was given by Jiang and Yau [6]. Specially, they showed that for a class of nice arrangement in $\mathbf{C P}^{2}$, the diffeomorphic types of the complements are combinatorial in nature. In this paper, we introduce a new class of simple arrangements in $\mathbf{C P}^{2}$. This class of simple arrangements is much larger than the class of nice arrangements. We prove that for this new class of simple arrangements, the diffeomorphic types of the complements are still combinatorial in nature. In fact, the moduli space of simple arrangements with fixed combinatorial data is connected.
\end{abstract}

\section{Introduction.}

An arrangement of hyperplanes is a finite collection of $\mathbf{C}$-linear subspaces of codimension one in a complex vector space $\mathbf{C}^{l}$. A central arrangement in $\mathbf{C}^{l}$ means that all hyperplanes of the arrangement pass through the origin. For such an arrangement $\mathcal{A}$, there is a natural projective arrangement $\mathcal{A}^{*}$ of hyperplanes in $\mathbf{C P}^{l-1}$ associated to it. Let $M(\mathcal{A})=\mathbf{C}^{l}-\bigcup_{H \in \mathcal{A}} H$ be a complement of $\mathcal{A}$, which is an open connected submanifold of $\mathbf{C}^{l}$. Let $M\left(\mathcal{A}^{*}\right)=\mathbf{C P}^{l-1}-\bigcup_{H^{*} \in \mathcal{A}^{*}} H^{*}$ be a complement of $\mathcal{A}^{*}$ in $\mathbf{C P}^{l}$.

There are many interesting research topics on the theory of arrangements (see $[1,9])$. One of the central topics is to find connection between

\footnotetext{
${ }^{1}$ Research partially supported by U.S. Army Research grant and NSF grant

${ }^{2}$ Ze-Jiang Professor of East China Normal University
} 
the topology or differentiable structure of $M(\mathcal{A})$ (or $\left.M\left(\mathcal{A}^{*}\right)\right)$ and the combinatorial geometry of $\mathcal{A}$. In 1980, Orlik and Solomon [10] gave a collection of 'homotopy type' conjectures, which assert that various homotopy invariants of the complement depend only on the lattice associated with $\mathcal{A}$. A great deal of research in the homotopy theory of arrangements has been focused on conjectures of this type. The major positive result in this direction was obtained by Jiang and Yau [6] in 1994.

Let $\mathcal{A}^{*}$ be a projective arrangement in $\mathbf{C P}^{2}$ and $L\left(\mathcal{A}^{*}\right)$ be the set of all intersections of elements of $\mathcal{A}^{*}$ partially ordered by inverse inclusion. It is natural to ask whether the combinatorial data $L\left(\mathcal{A}^{*}\right)$ of the projective arrangements is determined by the homotopy type, topological type, or diffeomorphic type of the complement $M\left(\mathcal{A}^{*}\right)$, and conversely whether the homotopy type, topological type, or diffeomorphic type of the complement $M\left(\mathcal{A}^{*}\right)$ of the projective arrangements is determined by the combinatorial data $L\left(\mathcal{A}^{*}\right)$. For the first question, Falk has written a series of papers $[2,3,4,5]$ on whether there are combinatorially distinct arrangements which have homotopic equivalent complements. In [4], Falk constructed two projective arrangements in $\mathbf{C P}^{2}$, each of which has two triple points and nine double points, but they are different combinatorially. The homotopic equivalence of their complements was shown in [5]. Jiang and Yau [8] showed that in general $L\left(\mathcal{A}^{*}\right)$ is a topological invariant of $M\left(\mathcal{A}^{*}\right)$, which is perhaps one of the deepest results in the subject. Hence, the two projective arrangements constructed by Falk do not have same topological types. For the second question, a partial solution was given by Jiang and Yau [6] in 1994 and [7] in 1997. They introduced a large class of arrangements in $\mathbf{C P}^{2}$ which are called nice arrangements, and showed that for these nice arrangements, the diffeomorphic types of the complement $M\left(\mathcal{A}^{*}\right)$ are determined by the combinatorial types of the arrangements. This paper generalizes the result of [6] to a much larger class of arrangements in $\mathbf{C P}^{2}$.

For a projective arrangement $\mathcal{A}^{*}$ in $\mathbf{C P}^{2}$, we can define a graph $G\left(\mathcal{A}^{*}\right)$ which depends only on the combinatorial data of the arrangement. A result of [8] asserts that $G\left(\mathcal{A}^{*}\right)$ depends only on the topological type of $M\left(\mathcal{A}^{*}\right)$. An arrangement $\mathcal{A}^{*}$ is called a simple arrangement if after removing pairwise disjoint stars (see Definition 2.3) and free simple nets of $G$ (see Definition 2.5), the graph $G$ from $\mathcal{A}^{*}$ becomes a forest. Recall that the concept of disjoint starts was introduced in [6]. The essential contribution of this paper is the new formulation of the concept of disjoint simple nets. This makes the class of simple arrangements a much larger class than the class of nice arrangements. 
Main Theorem 1.1. Let $\mathcal{A}_{0}$ and $\mathcal{A}_{1}$ be two simple arrangements in $\mathbf{C}^{3}$ and $\mathcal{A}_{0}^{*}$ and $\mathcal{A}_{1}^{*}$ be the corresponding projective arrangements in $\mathbf{C P}^{2}$. If the lattices of $\mathcal{A}_{0}$ and $\mathcal{A}_{1}$ are isomorphic, then $M\left(\mathcal{A}_{0}^{*}\right)$ and $M\left(\mathcal{A}_{1}^{*}\right)$ are diffeomorphic to each other. In fact, the moduli space of simple arrangements with fixed lattice is connected.

In Section 2, we introduce our new notion of free simple net from which we can define a new general class of simple arrangements. We also give some examples of simple arrangements. In Section 3, we correct some misprints of the lemmas in [6], prove the Main Theorem and give its corollary that the homotopy groups of the complement $M\left(\mathcal{A}^{*}\right)$ of a simple arrangement in $\mathbf{C P}^{2}$ depend only on the lattice of $\mathcal{A}$. We also define a class of generalized simple projective arrangements which includes the class of simple arrangements as a special case. We prove that the diffeomorphic types of the complements are still combinatorial in nature.

\section{Definitions and Examples.}

In this section, we denote $\mathcal{A}$ the (central) arrangement of hyperplanes in $\mathbf{C}^{\mathbf{3}}$ and $\mathcal{A}^{*}$ its associated projective arrangement of lines in $\mathbf{C P}^{\mathbf{2}}$. Let $L(\mathcal{A})$ be the lattice associated with $\mathcal{A}$.

Definition 2.1. A point $p$ in $\mathbf{C P}^{\mathbf{2}}$ is of multiplicity $k$ in $\mathcal{A}^{*}$ if $p$ is the intersection of exactly $k$ lines in $\mathcal{A}^{*}$. Let $t_{k}\left(\mathcal{A}^{*}\right)$ be the number of $k$-tuple points in the arrangement $\mathcal{A}^{*}$. The complexity $c\left(\mathcal{A}^{*}\right)$ of $\mathcal{A}^{*}$ is defined to be $\sum_{k \geq 3}(k-2) t_{k}\left(\mathcal{A}^{*}\right)$.

Now, we define a graph $G$ from an arrangement $\mathcal{A}^{*}$ in $\mathbf{C P}^{2}$. Let $V^{G}$ be the set of vertices of $G$ consisting of all points of $\mathcal{A}^{*}$ with multiplicity greater than 2. Let $E^{G}$ be the set of edges of $G$, each edge of which is a pair of distinct vertices $\left(v_{1}, v_{2}\right)$ of $V^{G}$ which span a line $<v_{1}, v_{2}>$ of $\mathcal{A}^{*}$.

Definition 2.2. A path of $G$ is denoted by a $(n+1)$-tuple $\left(v_{0}, \ldots, v_{n}\right)$ such that $\left(v_{i-1}, v_{i}\right) \in E^{G}$ for $i=1, \ldots, n-1$. Furthermore, it is called a circle when $v_{0}=v_{n}, n \geq 3$.

A reduced path is a path which satisfies $<v_{i-1}, v_{i}>\neq<v_{i}, v_{i+1}>$ for $i=1, \ldots, n-1$. If $v_{0}=v_{n}, n \geq 3$, then the reduced path is called a reduced circle. $G$ is called a forest if it does not contain such a reduced circle.

In this paper, we consider only reduced paths and reduced circles. 
Definition 2.3. For a $v_{0} \in V^{G}$, a star of $v_{0}$ is a subgraph of $G$, denoted by $S t\left(v_{0}\right)$, where $V^{S t\left(v_{0}\right)}=\left\{v_{0}\right\} \cup\left\{v \in V^{G}:<v_{0}, v>\in \mathcal{A}^{*}\right\}$ and $E^{S t\left(v_{0}\right)}=$ $\left\{(v, w) \in E^{G}: v=v_{0}\right.$ or $w=v_{0}$, otherwise $\left.<v, w>=<v_{0}, v>\right\}$.

Definition 2.4. ([6]) An arrangement $\mathcal{A}^{*}$ in $\mathbf{C P}^{\mathbf{2}}$ is called nice if the graph $G$ from $\mathcal{A}^{*}$ has the following property: There are $v_{1}, \ldots, v_{m} \in V^{G}$ such that $S t\left(v_{1}\right), \ldots, S t\left(v_{m}\right)$ are pairwise disjoint in $G$ and $G^{\prime}=G-\bigcup_{i=1}^{m}\left(E^{S t\left(v_{i}\right)} \cup\right.$ $\left.\left\{v_{i}\right\}\right)$ is a forest.

Now, we define the simple net and simple arrangement.

Definition 2.5. (a) For a reduced circle $B$ and another reduced circle or a vertex $C$, a net of $B$ and $C$ is a subgraph of $G$, denoted by $\operatorname{Net}(B, C)$, where

$$
\begin{gathered}
V^{N e t(B, C)}=V^{B} \cup V^{C} \cup N, \\
E^{N e t(B, C)}=\left\{(v, u) \in E^{G} \mid v, u \in V^{N e t(B, C)}\right\},
\end{gathered}
$$

where $N=\left\{v \in V^{G}-\left(V^{B} \cup V^{C}\right) \mid v\right.$ is connected with only one vertex in $V^{B} \cup V^{C}$ by an edge in $\left.E^{G}\right\}$.

$C, B$ and $N$ are called center, base and node of the net respectively.

(b) If any two non-adjacent vertices of center $C$ or two non-adjacent vertices of base $B$ are not connected by an edge, any two vertices of center $C$ do not connect a same vertex of the base $B$ by two edges, moreover, any two vertices of net do not connect a same vertex in $V^{G}-V^{N e t(B, C)}$ by two edges, then we call $N e t(B, C)$ simple net.

(c) A vertex $v \in V^{B}$ is called free in $G$ if there is no edge connecting $v$ and center. The simple net with free vertex is called free simple net.

Definition 2.6. An arrangement $\mathcal{A}^{*}$ in $\mathbf{C P}^{\mathbf{2}}$ is called simple if the graph $G$ from $\mathcal{A}^{*}$ has the following property: There are finitely many stars and free simple nets, say $\operatorname{St}\left(v_{1}\right), \ldots, S t\left(v_{m}\right)$ and $\operatorname{Net}\left(B_{1}, C_{1}\right), \ldots, N e t\left(B_{n}, C_{n}\right)$, which are pairwise disjoint in $G$ and

$$
\begin{aligned}
G^{\prime}=G- & \left\{\left(\bigcup_{i=1}^{m}\left(E^{S t\left(v_{i}\right)} \cup\left\{v_{i}\right\}\right)\right)\right. \\
& \left.\cup\left(\bigcup_{j=1}^{n}\left(E^{N e t\left(B_{j}, C_{j}\right)} \cup\left\{v \mid v \in V^{\operatorname{Net}\left(B_{j}, C_{j}\right)}-N_{j}\right\}\right)\right)\right\}
\end{aligned}
$$

is a forest, where $N_{j}$ is the node of the net $\operatorname{Net}\left(B_{j}, C_{j}\right), j=1, \ldots n$.

Clearly, a nice graph is simple. 
Example 2.7. The following graphs show the examples of free simple net and non-simple net. In Fig. 1

$$
\begin{aligned}
& C=\left(v_{1}, v_{2}, v_{3}, v_{4}, v_{1}\right) . \\
& B=\left(v_{5}, v_{6}, v_{7}, v_{8}, v_{9}, v_{5}\right) . \\
& \operatorname{Net}(B, C)=\left\{v_{1}, v_{2}, v_{3}, v_{4}, v_{5}, v_{6}, v_{7}, v_{8}, v_{9}, v_{10}, v_{11}, v_{12}\right\} \\
& \cup\left\{\left(v_{1}, v_{2}\right),\left(v_{2}, v_{3}\right),\left(v_{3}, v_{4}\right),\left(v_{4}, v_{1}\right),\left(v_{5}, v_{6}\right),\left(v_{6}, v_{7}\right),\right. \\
&\left(v_{7}, v_{8}\right),\left(v_{8}, v_{9}\right),\left(v_{9}, v_{5}\right),\left(v_{1}, v_{5}\right),\left(v_{2}, v_{6}\right),\left(v_{3}, v_{7}\right),\left(v_{4}, v_{9}\right), \\
&\left.\left(v_{5}, v_{10}\right),\left(v_{6}, v_{11}\right),\left(v_{8}, v_{12}\right)\right\} .
\end{aligned}
$$

$v_{8}$ is a free vertex. $\operatorname{Net}(B, C)$ is a free simple net.

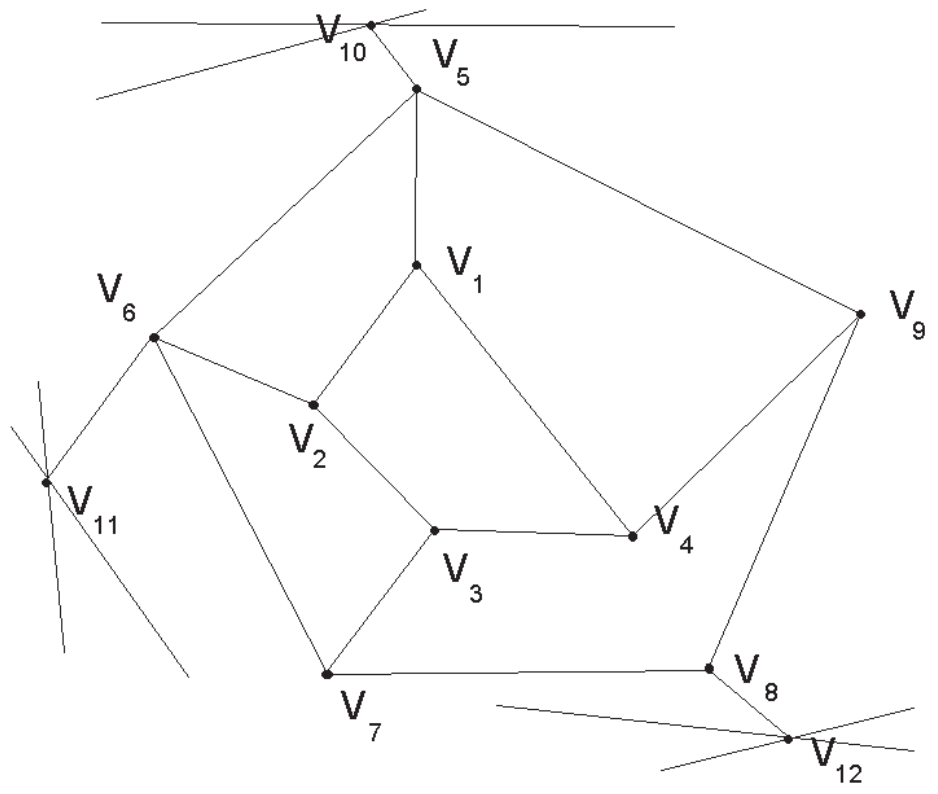

Figure 1: A free simple net

In Fig. 2, $\operatorname{Net}(B, C)$ is a non-simple net because the vertices $v_{6}$ and $v_{7}$ in $\operatorname{Net}(B, C)$ connect same vertex $v_{11}$ by edges $\left(v_{6}, v_{11}\right)$ and $\left(v_{7}, v_{11}\right)$ and $v_{5}$ in $B$ connects two vertices $v_{1}$ and $v_{2}$ in $C$ by two edges $\left(v_{1}, v_{5}\right)$ and $\left(v_{2}, v_{5}\right)$. 


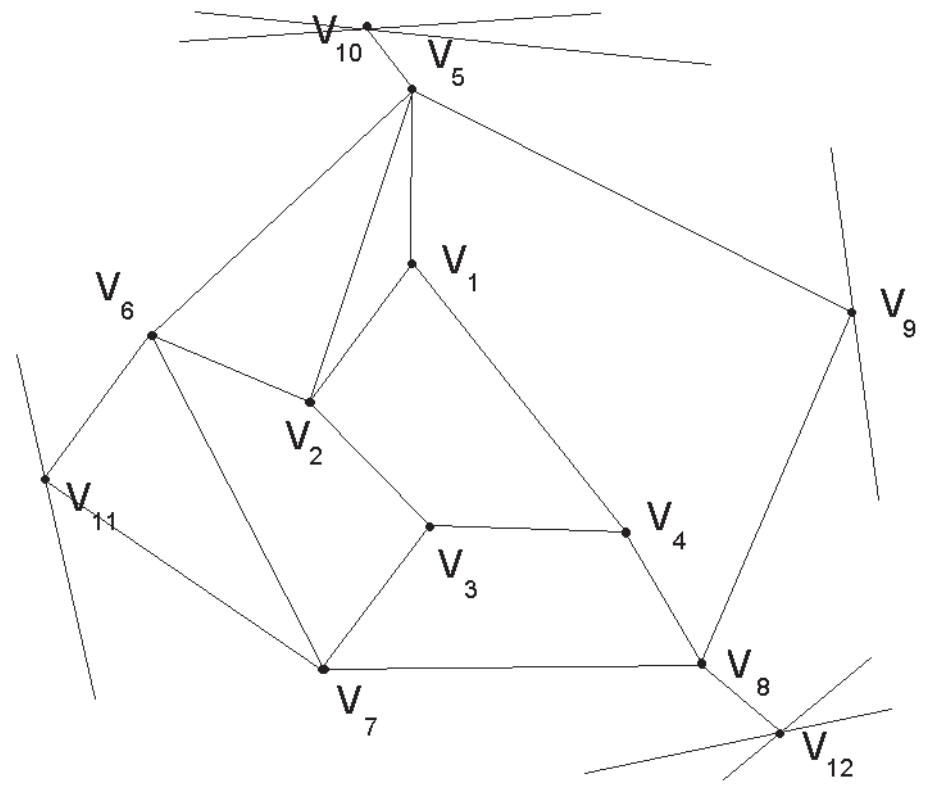

Figure 2: A non-simple net

Example 2.8. Let $\mathcal{A}$ be an arrangement of hyperplanes in $\mathbf{C}^{\mathbf{3}}$ and $\mathcal{A}^{*}$ associated arrangement in $\mathbf{C P}^{\mathbf{2}}$ as shown in the following figures. This is an example of simple arrangement and it is not a nice arrangement. In Figs. 3,4 and 5 ,

$$
\begin{aligned}
\operatorname{St}\left(v_{1}\right)= & \left\{v_{1}, v_{6}, v_{7}, v_{9}\right\} \cup\left\{\left(v_{1}, v_{6}\right),\left(v_{1}, v_{7}\right),\left(v_{1}, v_{9}\right)\right\} . \\
C= & \left\{v_{3}\right\} . \\
B= & \left(v_{2}, v_{4}, v_{8}, v_{5}, v_{2}\right) . \\
\operatorname{Net}(B, C)= & \left\{v_{2}, v_{3}, v_{4}, v_{5}, v_{8}, v_{11}\right\} \\
\cup & \left\{\left(v_{3}, v_{2}\right),\left(v_{3}, v_{4}\right),\left(v_{3}, v_{8}\right),\left(v_{2}, v_{4}\right),\left(v_{2}, v_{5}\right),\left(v_{4}, v_{8}\right),\right. \\
& \left(v_{5}, v_{8}\right),\left(v_{8}, v_{11}\right\} .
\end{aligned}
$$

$v_{5}$ is free vertex. $\operatorname{Net}(B, C)$ is a free simple net. $\mathcal{A}^{*}$ is a simple arrangement, but not a nice arrangement. 
Rigidity of Differentiable Structure for New Class of Line Arrangements1063

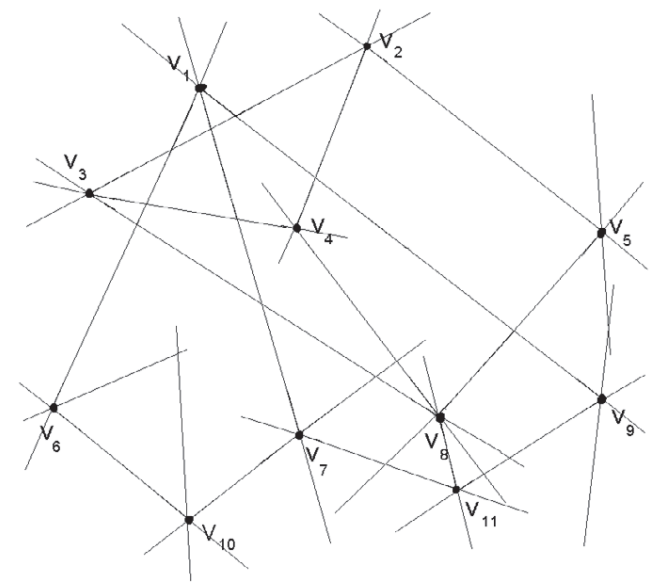

Figure 3: A simple arrangement $\mathcal{A}^{*}$ including the line in infinite

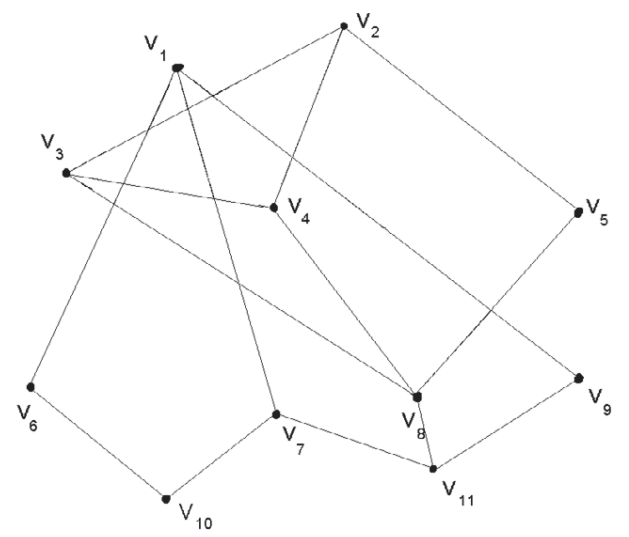

Figure 4: The graph $G$ of $\mathcal{A}^{*}$

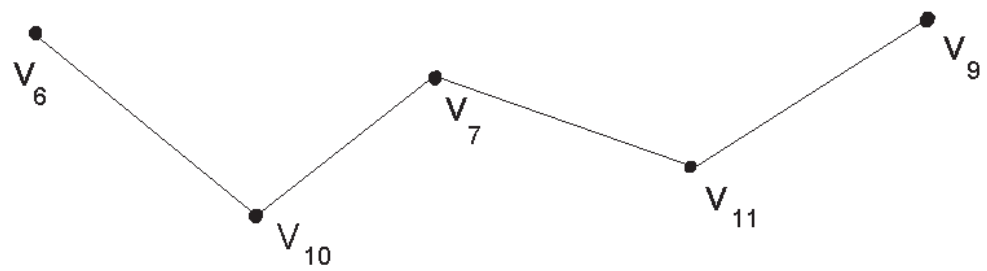

Figure 5: The graph $G^{\prime}$ 


\section{Proof of the Main Theorem.}

The following Definition 3.1 to Remark 3.5 can be found in [6]. Since they play an important role in the proof of our main theorem and there are some misprints in [6], for the sake of convenience to the readers, we include them below.

Definition 3.1. For the following equation

$$
a y_{1} x_{2} x_{3}+b x_{1} y_{2} x_{3}+c x_{1} x_{2} y_{3}+d x_{1} y_{2} y_{3}+e y_{1} x_{2} y_{3}+f y_{1} y_{2} x_{3}=0
$$

where $\left(x_{1}: y_{1}\right),\left(x_{2}: y_{2}\right)$ and $\left(x_{3}: y_{3}\right) \in \mathbf{C P}^{1}$ are different variables, $a, b, c, d, e$ and $f \in \mathbf{C}$, and abcdef $\neq 0,\left(x_{1}: y_{1}\right)$ is called irregular for Equation (3.1) if

$$
\left(a y_{1}\right) x_{2} x_{3}+\left(b x_{1}+f y_{1}\right) y_{2} x_{3}+\left(c x_{1}+e y_{1}\right) x_{2} y_{3}+\left(d x_{1}\right) y_{2} y_{3}
$$

is a reducible polynomial of the other two variables $\left(x_{2}: y_{2}\right)$ and $\left(x_{3}: y_{3}\right)$. Otherwise, we call $\left(x_{1}: y_{1}\right)$ regular for the Equation (3.1).

Lemma 3.2. Assume $\left(\left(x_{1}: y_{1}\right),\left(x_{2}: y_{2}\right),\left(x_{3}: y_{3}\right)\right) \in\left(\mathbf{C P}^{1}\right)^{3}$ is a solution of (3.1). If $\left(x_{1}: y_{1}\right)$ is irregular, then either $\left(x_{2}: y_{2}\right)$ or $\left(x_{3}: y_{3}\right)$ is irregular for (3.1). If $\left(x_{1}: y_{1}\right)$ is regular, then $\left(x_{2}: y_{2}\right)$ and $\left(x_{3}: y_{3}\right)$ are either both regular or both irregular for (3.1).

Proof. When $y_{1}=0,(3.1)$ becomes

$$
b y_{2} x_{3}+c x_{2} y_{3}+d y_{2} y_{3}=0
$$

which is irreducible. Hence, if $\left(x_{1}: y_{1}\right)$ is irregular, then $x_{1} \neq 0$ and $y_{1} \neq 0$.

Write (3.1) as polynomial of $\left(x_{2}: y_{2}\right)$ and $\left(x_{3}: y_{3}\right)$

$$
\left(a y_{1}\right) x_{2} x_{3}+\left(b x_{1}+f y_{1}\right) y_{2} x_{3}+\left(c x_{1}+e y_{1}\right) x_{2} y_{3}+\left(d x_{1}\right) y_{2} y_{3}=0 .
$$

It is reducible if and only if

$$
\left(b x_{1}+f y_{1}\right)\left(c x_{1}+e y_{1}\right)=a d x_{1} y_{1}
$$

or

$$
b c x_{1}^{2}+(b e+f c-a d) x_{1} y_{1}+e f y_{1}^{2}=0
$$


Rigidity of Differentiable Structure for New Class of Line Arrangements1065

which has at most two roots of $\left(x_{1}: y_{1}\right)$. When $\left(x_{1}: y_{1}\right)$ is a root of the equation above, (3.1) becomes

$$
\left[\left(a y_{1}\right) x_{3}+\left(c x_{1}+e y_{1}\right)\right]\left[x_{2}+\frac{d x_{1}}{c x_{1}+e y_{1}} y_{2}\right]=0
$$

from which we have the solution either

$$
\left(x_{2}: y_{2}\right)=\left(-d x_{1}: c x_{1}+e y_{1}\right)
$$

or

$$
\left(x_{3}: y_{3}\right)=\left(-\left(c x_{1}+e y_{1}\right): a y_{1}\right) .
$$

In the first case, we have

$$
\frac{x_{1}}{y_{1}}=-\frac{e x_{2}}{c x_{2}+d y_{2}} .
$$

Using these in (3.5) yields

$$
b c \frac{e^{2} x_{2}^{2}}{\left(c x_{2}+d y_{2}\right)^{2}}-(b e+f c-d a) \frac{e x_{2}}{\left(c x_{2}+d y_{2}\right)}+e f=0 .
$$

Combining the like terms, we get

$$
b c e x_{2}^{2}-(b e+f c-d a)\left(c x_{2}^{2}+d x_{2} y_{2}\right)+\left(f c^{2} x_{2}^{2}+2 c d f x_{2} y_{2}+f d^{2} y_{2}^{2}\right)=0
$$

$$
c a x_{2}^{2}+(a d+f c-b e) x_{2} y_{2}+f d y_{2}^{2}=0 .
$$

The last equation (3.8) is a necessary and sufficient condition for $\left(x_{2}: y_{2}\right)$ being irregular of (3.1).

For the second case, we have the same conclusion for $\left(x_{3}: y_{3}\right)$.

From the argument above, we also have

Lemma 3.3. There are at most two irregular $\left(x_{i}: y_{i}\right)$ of (3.1) for each $i=1,2,3$. (0:1) and (1:0) are regular of (3.1).

Lemma 3.4. For each fixed regular $\left(x_{1}: y_{1}\right)$ of (3.1), the following relation produces an automorphism of $\mathbf{C P}^{1}$

$$
\left(\begin{array}{l}
x_{3} \\
y_{3}
\end{array}\right)=K\left(\begin{array}{cc}
-e y_{1}-c x_{1} & -d x_{1} \\
a y_{1} & b x_{1}+f y_{1}
\end{array}\right)\left(\begin{array}{l}
x_{2} \\
y_{2}
\end{array}\right), K \in \mathbf{C}^{*}
$$

which sends regular values to regular values of (3.1). In particular, $\left(x_{1}: y_{1}\right)=\left(x_{2}: y_{2}\right)=(0: 1)$ (respectively $\left.(1: 0)\right)$ corresponds to $\left(x_{3}: y_{3}\right)=$ $(0: 1)$ (respectively $(1: 0))$. 
Proof. Consider

$$
\left|\begin{array}{cc}
-e y_{1}-c x_{1} & -d x_{1} \\
a y_{1} & b x_{1}+f y_{1}
\end{array}\right|=-b c x_{1}^{2}-(b e+f c-d a) x_{1} y_{1}-f e y_{1}^{2} .
$$

Since $\left(x_{1}: y_{1}\right)$ is a regular value, the above expression is non-zero by (3.5). Hence, (3.9) is an automorphism of $\mathbf{C P}^{1}$. Clearly, (3.9) satisfies Equation (3.1). By Lemma 3.1, the mapping (3.9) sends regular values of (3.1) to regular values of (3.1). The last statement of the lemma is obvious.

Remark 3.5. Equation (3.9) is equivalent to Equation (3.1).

If we write (3.1) as

$$
\left(a y_{1} x_{2}+b x_{1} y_{2}+f y_{1} y_{2}\right) x_{3}+\left(d x_{1} y_{2}+e y_{1} x_{2}+c x_{1} x_{2}\right) y_{3}=0 .
$$

Then $\left(x_{3}, y_{3}\right)=K\left(-d x_{1} y_{2}-e y_{1} x_{2}-c x_{1} x_{2}, a y_{1} x_{2}+b x_{1} y_{2}+f y_{1} y_{2}\right)$ which is (3.9). Hence, if $\left(x_{1}: y_{1}\right)$ and $\left(x_{2}: y_{2}\right)$ are regular of (3.1), then there is a unique $\left(x_{3}: y_{3}\right)$ solved in terms of $\left(x_{1}: y_{1}\right)$ and $\left(x_{2}: y_{2}\right)$. We call such a procedure "fixing two variables to solve the other" and call $\left(x_{1}: y_{1}\right),\left(x_{2}: y_{2}\right),\left(x_{3}: y_{3}\right)$ "solved variables".

Definition 3.6. Let $G$ be a graph from a simple arrangement and $T$ be a connected component in $G^{\prime}$ which is a forest. Then $T$ is a tree. We call a vertex of $T$ an end vertex if it is also a vertex of a star or free simple net.

A length of the reduced path $P$ from vertex $v_{1}$ to $v_{2}$ is the number of the edges in $P$. The distance from $v_{1}$ to $v_{2}$ is the smallest length of the reduced path among all reduced paths from $v_{1}$ to $v_{2}$. The closest vertex different from $v_{1}$ to $v_{1}$ is the vertex whose distance from $v_{1}$ is the smallest among all vertices connecting $v_{1}$ by reduced paths.

Lemma 3.7. Let $G$ be a graph from a simple arrangement and $T$ be a tree in $G^{\prime}$. Then

(a) Each end vertex of $T$ connects only one edge of star or free simple net.

(b) For any two vertices of $T$, there is exactly one reduced path connecting them.

Proof. (a) is clear since the stars and free simple nets are pairwise disjoint.

Since $T$ is a tree, there is a path connecting any two vertices. If there are two reduced paths connecting two vertices, then these two reduced paths contain a circle. It is a contradiction. Hence, (b) is true. 
Rigidity of Differentiable Structure for New Class of Line Arrangements1067

Lemma 3.8. (Lattice-Isotopy Theorem [12]) If two arrangements are connected by a one-parameter family of arrangements which have the same lattice, the complements are diffeomorphic, hence of the same homotopy type.

Now, we state and prove our main theorem.

Main Theorem 3.9. Let $\mathcal{A}_{0}^{*}$ and $\mathcal{A}_{1}^{*}$ be two simple projective arrangements in $\mathbf{C P}^{2}$. If the lattices of $\mathcal{A}_{0}$ and $\mathcal{A}_{1}$ are isomorphic, then the complements $M\left(\mathcal{A}_{0}^{*}\right)$ and $M\left(\mathcal{A}_{1}^{*}\right)$ are diffeomorphic to each other.

Proof. We represent the two arrangements as $\mathcal{A}_{1}^{*}=\left\{H_{1}, H_{2}, \ldots, H_{n}\right\}$ and $\mathcal{A}_{0}^{*}=\left\{G_{1}, G_{2}, \ldots, G_{n}\right\}$ where $H_{i}=\left(h_{i 1}, h_{i 2}, h_{i 3}\right)$ and $G_{i}=\left(g_{i 1}, g_{i 2}, g_{i 3}\right)$ (Here, we use the coefficient vector of the defining equation to represent the hyperplane.) are in $\mathbf{C P}^{\mathbf{2}}$. We shall construct a one-parameter family of arrangements $\mathcal{A}^{*}(t)$ such that $\mathcal{A}^{*}(0)=\mathcal{A}_{0}^{*}, \mathcal{A}^{*}(1)=\mathcal{A}_{1}^{*}$ and $L(\mathcal{A}(t)) \equiv$ $L\left(\mathcal{A}_{0}\right)$ for all $t \in[0,1]$.

Let $\mathcal{A}^{*}=\left\{F_{1}, F_{2}, \ldots, F_{n}\right\}$ where $F_{i}=x_{i} G_{i}+y_{i} H_{i}$ for some $x_{i}, y_{i} \in \mathbf{C}$ such that $F_{i}$ is in $\mathbf{C P}^{2}, i=1,2, \ldots, n$. Let $I=\{(i, j, k): 1 \leq i<j<k \leq$ $n\}$. So $|I|=\left(\begin{array}{l}n \\ 3\end{array}\right)$. Consider any triple $\left(F_{i}, F_{j}, F_{k}\right),(i, j, k) \in I$. Denote the matrix

$$
\left(\begin{array}{ccc}
x_{i} g_{i 1}+y_{i} h_{i 1} & x_{i} g_{i 2}+y_{i} h_{i 2} & x_{i} g_{i 3}+y_{i} h_{i 3} \\
x_{j} g_{j 1}+y_{j} h_{j 1} & x_{j} g_{j 2}+y_{j} h_{j 2} & x_{j} g_{j 3}+y_{j} h_{j 3} \\
x_{k} g_{k 1}+y_{k} h_{k 1} & x_{k} g_{k 2}+y_{k} h_{k 2} & x_{k} g_{k 3}+y_{k} h_{k 3}
\end{array}\right)
$$

by $\left[F_{i} F_{j} F_{k}\right]$ and its determinant by $\left|F_{i} F_{j} F_{k}\right|$. We now can write

$$
\begin{aligned}
\left|F_{i} F_{j} F_{k}\right|= & \left|G_{i} G_{j} G_{k}\right| x_{i} x_{j} x_{k}+\left|H_{i} G_{j} G_{k}\right| y_{i} x_{j} x_{k}+\left|G_{i} H_{j} G_{k}\right| x_{i} y_{j} x_{k} \\
& +\left|G_{i} G_{j} H_{k}\right| x_{i} x_{j} y_{k}+\left|G_{i} H_{j} H_{k}\right| x_{i} y_{j} y_{k}+\left|H_{i} G_{j} H_{k}\right| y_{i} x_{j} y_{k} \\
& +\left|H_{i} H_{j} G_{k}\right| y_{i} y_{j} x_{k}+\left|H_{i} H_{j} H_{k}\right| y_{i} y_{j} y_{k} .
\end{aligned}
$$

Since two distinct lines in $\mathbf{C P}^{2}$ meet exactly at one point, to get $L(\mathcal{A}) \equiv$ $L\left(\mathcal{A}_{0}\right)$, it is sufficient to have the following: For any $(i, j, k) \in I$,

$$
\left|F_{i} F_{j} F_{k}\right|=0 \text { if and only if }\left|G_{i} G_{j} G_{k}\right|=0 .
$$

Let $l=\sum_{j \geq 3}\left(\begin{array}{l}j \\ 3\end{array}\right) t_{j}\left(\mathcal{A}_{1}^{*}\right)$, we need to have $l$ equations and $\left(\begin{array}{l}n \\ 3\end{array}\right)-l$ inequalities

$$
\begin{gathered}
P_{1}=0, \ldots, P_{l}=0 . \\
Q_{1} \neq 0, \ldots, Q_{\left(\begin{array}{c}
n \\
3
\end{array}\right)-l} \neq 0 .
\end{gathered}
$$


Both $P_{i}$ and $Q_{j}$ have the forms like (3.10). But for $P_{i}$, the first term and last term are zero since $\left|G_{i} G_{j} G_{k}\right|=\left|H_{i} H_{j} H_{k}\right|=0$ by (3.11). Among $P_{1}, \ldots, P_{l}$ at most $c\left(\mathcal{A}_{1}^{*}\right)=\sum_{j \geq 3}(j-2) t_{j}\left(\mathcal{A}_{1}^{*}\right)$ of them are independent. To see this, we consider a $j$-tuple point $v(j \geq 3)$. Let $F_{1}, \ldots, F_{j}$ be the lines of $\mathcal{A}^{*}$ passing though $v$. We have $\left(\begin{array}{l}j \\ 3\end{array}\right)$ equations $\left(\left|F_{i} F_{j} F_{k}\right|=0, \ldots\right.$, etc. $)$. Since $\left\{F_{1}, \ldots, F_{j}\right\}$ can be linearly generated by $F_{1}$ and $F_{2}$, the $\left(\begin{array}{l}j \\ 3\end{array}\right)$ equations are reduced equivalently to $j-2$ equations $\left|F_{1} F_{2} F_{k}\right|=0$ for $i=3, \ldots, j$. Now, consider all $j$-tuple points $(j \geq 3)$. We have a system of $c\left(\mathcal{A}_{1}^{*}\right)$ equations, say $\left\{P_{1}=0, \ldots, P_{c\left(\mathcal{A}_{1}^{*}\right)}=0\right\}$ which is equivalent to $\left\{P_{1}=0, \ldots, P_{l}=0\right\}$.

As we observed before, each $P_{r}$ can be written as

$$
\begin{aligned}
P_{r}= & a_{r} y_{i_{r}} x_{j_{r}} x_{k_{r}}+b_{r} x_{i_{r}} y_{j_{r}} x_{k_{r}}+c_{r} x_{i_{r}} x_{j_{r}} y_{k_{r}}+d_{r} x_{i_{r}} y_{j_{r}} y_{k_{r}}+e_{r} y_{i_{r}} x_{j_{r}} y_{k_{r}} \\
& +f_{r} y_{i_{r}} y_{j_{r}} x_{k_{r}}
\end{aligned}
$$

where $a_{r}=\left|H_{i r} G_{j r} G_{k r}\right|$ etc. Replacing $\mathcal{A}^{*}$ by $\phi\left(\mathcal{A}^{*}\right)$ if necessary where $\phi: \mathbf{C P}^{2} \rightarrow \mathbf{C P}^{2}$ is a complex analytic automorphism, we assume without loss of generality that any one (two) line(s) in $\mathcal{A}_{0}^{*}$ and any two (one) line(s) in $\mathcal{A}_{1}^{*}$ do not intersect at a point. This means that $a_{r} b_{r} c_{r} d_{r} e_{r} f_{r} \neq 0$ for all $r=1, \ldots, c\left(\mathcal{A}_{1}^{*}\right)$.

Note that $P_{r}$ is viewed as a polynomial in $\left(\left(x_{1}: y_{1}\right), \ldots,\left(x_{n}: y_{n}\right)\right) \in$ $\left(\mathbf{C P}^{\mathbf{1}}\right)^{n}$. For each $r$, indices $i_{r}, j_{r}, k_{r}$ are pairwise distinct and $\left(i_{r}, j_{r}, k_{r}\right) \neq$ $\left(i_{s}, j_{s}, k_{s}\right)$ for $r \neq s$ where $1 \leq i_{r}, j_{r}, k_{r}, i_{s}, j_{s}, k_{s} \leq n$ and $1 \leq r, s \leq c\left(\mathcal{A}_{1}^{*}\right)$.

Since $\mathcal{A}_{1}^{*}$ is a simple projective arrangement in $\mathbf{C P}^{2}$, there are the disjoint stars and simple nets, say $S t\left(v_{1}\right), \ldots, S t\left(v_{m}\right)$ and $\operatorname{Net}\left(B_{1}, C_{1}\right), \ldots, N e t\left(B_{n}, C_{n}\right)$ in $G$ such that

$$
\begin{aligned}
& G^{\prime}=G-\left\{( \bigcup _ { i = 1 } ^ { m } ( E ^ { S t ( v _ { i } ) } \cup \{ v _ { i } \} ) ) \cup \left(\bigcup _ { j = 1 } ^ { n } \left(E^{N e t\left(B_{j}, C_{j}\right)}\right.\right.\right. \\
&\left.\left.\left.\cup\left\{v \mid v \in V^{N e t\left(B_{j}, C_{j}\right)}-N_{j}\right\}\right)\right)\right\}
\end{aligned}
$$

is a forest, where $N_{j}$ is the node of the net $\operatorname{Net}\left(B_{j}, C_{j}\right), j=1, \ldots n$.

We shall prove that we can solve all variables in terms of some variables (in the sense of Remark 3.5) without ambiguity. Here, we shall use the notation in Definition 2.6.

Case 1: Suppose $m=1, n=0$, i.e. there is only a star $S t(v)$.

Assume that $v$ is a vertex of multiplicity $k$ in $\mathcal{A}_{1}^{*}$. Since $k \geq 3$ by definition of $G$, there are $k$ variables appearing in $k-2$ equations of 
(3.14). Without loss of generality, we suppose that these variables are $\left(x_{1}: y_{1}\right), \ldots,\left(x_{k}: y_{k}\right)$ and $\left(x_{1}: y_{1}\right)$ and $\left(x_{2}: y_{2}\right)$ appear in each of these $k-2$ equations. Thus, we can fix $\left(x_{1}: y_{1}\right)$ and $\left(x_{2}: y_{2}\right)$ to solve $\left(x_{3}: y_{3}\right), \ldots,\left(x_{k}: y_{k}\right)$.

The rest of the unsolved variables and equations in (3.14) correspond to the graph $G^{\prime}$ which is a forest. A connected component $T$ is a tree of $G^{\prime}$. We consider these trees respectively. Assume that the end vertices of $T$ are $v_{1}, v_{2}, \ldots, v_{t}$.

If $t=1$, that is, there is only one end vertex, say $v_{1}$, which is also the vertex of the edge $E$ in the star. By Lemma 3.7, $v_{1}$ only connects $E$ in star. We fix the variable corresponding to $E$ and any another variable corresponding to the edge other than $E$ issuing from $v_{1}$. Then we can solve all variables about the tree by fixing these two variables.

If $t \geq 2$, we choose any end vertex, say $v_{1}$. From Lemma 3.7, $v_{1}$ connects only one edge $E$ of the star and there is only one reduced path from $v_{1}$ to another end vertex. We choose the closest end vertex from $v_{1}$, say $v_{2}$. Assume that $P_{1}$ is this reduced path connecting $v_{1}$ and $v_{2}$. Along this reduced path from $v_{1}$ to $v_{2}$, we can fix the variable corresponding to the edge $E$ of star and any other variable corresponding to the edge issuing from $v_{1}$ on $P_{1}$ and solve other variables corresponding to the edges on $P_{1}$. At $v_{2}$, there are only two edges (one from the star, one from the path $P_{1}$ ) whose variables are solved. Hence, we can fix these two solved variables to solve others corresponding to the edges issuing from $v_{2}$. Then we consider other end vertices not in $P_{1}$. Pick one, say $v_{3}$, which is connected to a vertex on $P_{1}$, say $w_{1}$. Assume a path $P_{2}$ is a shortest path which connects $w_{1}$ and $v_{3}$. At $w_{1}$, there are only two edges from $P_{1}$ or star whose variables are solved. We use these two solved variables to solve other variables corresponding to the edges along the path $P_{2}$ from $w_{1}$ to $v_{3}$. At $v_{3}$, by Lemma $3.7, v_{3}$ connects only one edge in star and one edge in $P_{2}$ whose variables are solved. Fixing the variables corresponding to these two edges, we can solve other variables corresponding to the edges issuing from $v_{3}$. Now, we consider other end vertices not in $P_{1}$ and $P_{2}$. Pick one, say $v_{4}$, which is connected to a vertex on $P_{1} \cup P_{2}$, say $w_{2}$. Assume a path $P_{3}$ is a shortest path which connects $w_{2}$ and $v_{4}$. Similar to the above, we can solve other variables corresponding to the edges along the path $P_{3}$ from $w_{2}$ to $v_{4}$. We continue the same procedure until last end vertex, say $v_{t}$. Since $T$ is a tree, we can assume that there is only one path, say $P_{t-1}$, connecting some vertex, say $w_{t-2}$, on the path $P_{1} \cup P_{2} \cup \cdots \cup P_{t-2}$ and $v_{t}$. Otherwise, we will find a circle in $T$. Thus, at $w_{t-2}$, there are only two variables solved which correspond to the edges on $P_{1} \cup P_{2} \cup \cdots \cup P_{t-2}$ or in the star. We fix these two variables corresponding 
to these two edges and solve other variables along $P_{t-1}$. At $v_{t}$, we can fix two variables corresponding to the edge of star and other variable corresponding to the edge on $P_{t-1}$ and solve other variables. Thus, we can solve all variables in terms of some variables without ambiguity.

Case 2: Suppose $m=0$ and $n=1$, i.e. there is only a free simple net $\operatorname{Net}(B, C)$.

Without loss of generality, let $B,\left(v_{0}, v_{1}, \ldots, v_{q}\right),\left(v_{q}=v_{0}\right)$, be the circle with a free vertex $v_{0}$.

If $C$ is a vertex $v$ of multiplicity $k$ in $\mathcal{A}_{1}^{*}$. Since $k \geq 3$ by definition of $G$, there are $k$ variables appearing in $k-2$ equations of (3.14) corresponding to $v$. We can fix any two variables, say $\left(x_{1}: y_{1}\right)$ and $\left(x_{2}: y_{2}\right)$, to solve the other variable $\left(x_{3}: y_{3}\right), \ldots,\left(x_{k}: y_{k}\right)$ corresponding to $v$.

Now, we consider base $B$. If each vertex on $B$ is free, then there is not any edge between $B$ and $v$. Choosing any vertex on $B$, say $v_{i}$. We fix any two variables corresponding to the edges issuing from $v_{i}$ and solve the other variables related. Next step, we consider $v_{i+1}$ (or $v_{i-1}$ ). Since $v_{i+1}$ ( or $v_{i-1}$ ) is free, there is only one solved variable corresponding to the edge $\left(v_{i}, v_{i+1}\right)$ (or $\left.\left(v_{i}, v_{i-1}\right)\right)$ issuing from $v_{i+1}$ (or $\left.v_{i-1}\right)$. We use this solved variable and fix any other variable to solve all other variables corresponding to the edges issuing from $v_{i+1}$ (or $v_{i-1}$ ). Continuing this step till last vertex, say $v_{q}\left(=v_{0}\right)$. Since $v_{q}$ is free, there are only two edges $\left(v_{q}, v_{q-1}\right)$ and $\left(v_{0}, v_{1}\right)$ whose variables are solved. We use these two variables to solve all other variables at $v_{q}$.

If there is a non-free vertex on $B$, say $v_{i},(i \neq q$ and 0$)$. Assume the multiplicity of $v_{i}$ is $t(\geq 3)$. Similarly, there are $t$ variables appearing in $t-2$ equations of (3.14) corresponding to $v_{i}$. Since $v_{i}$ is adjacent to $v$, the variable corresponding to the edge $\left(v, v_{i}\right)$ is solved, we can use the solved variable, say, $\left(x_{1}: y_{1}\right)$ and fix any other variable, say, $\left(x_{k+1}: y_{k+1}\right)$ to solve all other variables $\left(x_{k+2}: y_{k+2}\right), \ldots,\left(x_{k+t-1}: y_{k+t-1}\right)$ at $v_{i}$. At the following step, we choose vertex $v_{i+1}$ (or $v_{i-1}$ ) along the circle $B$. If $v_{i+1}$ (or $v_{i-1}$ ) is free, then there is only one solved variable corresponding to the edge $\left(v_{i}, v_{i+1}\right)$ (or $\left(v_{i}, v_{i-1}\right)$ ) issuing from $v_{i+1}$ (or $\left.v_{i-1}\right)$. We use this solved variable and fix any other variable to solve all other variables corresponding to the edges issuing from $v_{i+1}$ (or $v_{i-1}$ ). If $v_{i+1}$ ( or $v_{i-1}$ ) is not free, then there are only two edges $\left(v_{i}, v_{i+1}\right)$ and $\left(v, v_{i+1}\right)$ (or $\left(v_{i}, v_{i-1}\right)$ and $\left.\left(v, v_{i-1}\right)\right)$ whose variables are solved. We use these two solved variables corresponding to the edges $\left(v, v_{i+1}\right)$ and $\left(v_{i}, v_{i+1}\right)$ (or $\left(v, v_{i-1}\right)$ and $\left(v_{i}, v_{i-1}\right)$ respectively) to solve other variables corresponding to the edges issuing from $v_{i+1}$ (or $v_{i-1}$ ). We apply the same procedure till $v_{q}\left(v_{0}\right)$. Since $v_{0}$ is free, it does not connect $C$ by an edge. Hence, there are only two edges on $B$ passing through $v_{0}$. Thus, 
we can use the solved variables corresponding to these two edges $\left(v_{0}, v_{1}\right)$ and $\left(v_{q}, v_{q-1}\right)$ to solve the other variables corresponding to the edges issuing from $v_{0}$.

If $C$ is a circle, say, $\left(w_{0}, w_{1}, \ldots, w_{p}\right),\left(w_{p}=w_{0}\right)$. Let $k=$ maximum of multiplicities of all vertices in $C$. Choosing any vertex $w_{i}, i \neq 0, p$ with multiplicity $k(\geq 3)$ in $C$ (we can rerange the indices so that $i \neq 0, p$ ). Then there are $k$ variables appearing in $k-2$ equations of (3.14) corresponding to $w_{i}$. Without loss of generality, we suppose that $\left(x_{1}: y_{1}\right)$ and $\left(x_{2}: y_{2}\right)$ appear in each of these $k-2$ equations. We can fix $\left(x_{1}: y_{1}\right)$ and $\left(x_{2}: y_{2}\right)$ to solve the other variables $\left(x_{3}: y_{3}\right), \ldots,\left(x_{k}: y_{k}\right)$. At next step, we choose the vertex $w_{i+1}$ (or $w_{i-1}$ ) along $C$ and use one solved variable from $\left(w_{i}, w_{i+1}\right)$ (or $\left(w_{i}, w_{i-1}\right)$ ) and fix any other variable corresponding to the edge issuing from $w_{i+1}$ or $\left(w_{i-1}\right)$ to solve the variables corresponding to the edges issuing from $w_{i+1}\left(\right.$ or $\left.w_{i-1}\right)$. We apply same procedure till $w_{p}\left(w_{0}\right)$. Since $C$ is a circle and $w_{p}=w_{0}$, there are only two edges $\left(w_{1}, w_{0}\right)$ and $\left(w_{p-1}, w_{p}\right)$ whose variables are solved at $w_{0}$. We use these two solved variables from $\left(w_{1}, w_{0}\right)$ and $\left(w_{p-1}, w_{p}\right)$ to solve the variables corresponding to the edges issuing from $w_{0}$.

Now, we consider base circle $B,\left(v_{0}, v_{1}, \ldots, v_{q}\right),\left(v_{q}=v_{0}\right)$, with a free vertex $v_{0}$. Since $\operatorname{Net}(B, C)$ is simple, there is at most one edge between a vertex $v$ of $B$ and $C$. If all vertices of $B$ are free, then there is not any edge between $B$ and $C$. In other words, $B$ and $C$ are not related. Notice that $B$ and $C$ are circles. Thus, we can solve the variables corresponding to the edges on $B$ is the same manner as solving the variables corresponding to the edges on $C$ as above. If there is some non-free vertex on $B$, choosing a non-free vertex, say $v_{i} \in B$, which is connected $C$ by one edge, say $\left(w_{j}, v_{i}\right)$, whose variable is solved, we use the solved variable from $\left(w_{j}, v_{i}\right)$ and fix any other variable to solve other variables corresponding to the edges issuing from $v_{i}$. At the following step, we choose vertex $v_{i+1}$ (or $v_{i-1}$ ) from $B$. If $v_{i+1}$ (or $v_{i-1}$ ) is free, then there is only one solved variable corresponding to the edge $\left(v_{i}, v_{i+1}\right)$ (or $\left(v_{i}, v_{i-1}\right)$ ) issuing from $v_{i+1}$ (or $\left.v_{i-1}\right)$. We use this solved variable and fix any other variable to solve all other variables corresponding to the edges issuing from $v_{i+1}$ (or $v_{i-1}$ ). If $v_{i+1}\left(\right.$ or $v_{i-1}$ ) is not free, since $\operatorname{Net}(B, C)$ is simple, there is only one edge connecting $v_{i+1}$ (or $v_{i-1}$ ) and some vertex in $C$, say $w_{t}$. Thus, there are only two variables solved corresponding to the edges $\left(v_{i+1}, v_{i}\right)$ and $\left(w_{t}, v_{i+1}\right)$ (or $\left(v_{i-1}, v_{i}\right)$ and $\left.\left(w_{t}, v_{i-1}\right)\right)$. We use these two solved variables to solve other variables issuing from $v_{i+1}$ (or $\left.v_{i-1}\right)$. We apply the same procedure till $v_{q}\left(v_{0}\right)$. Last step, we solved variables about free vertex $v_{0}$ where there are only two vertices $v_{1}$ and $v_{q-1}$ connecting it in $B$. Hence, We can use these two solved variables 
from $\left(v_{1}, v_{0}\right)$ and $\left(v_{q-1}, v_{q}\right)$ to solve the variables corresponding to the edges issuing from $v_{0}$.

The rest of the unsolved variables and equations in (3.14) correspond to the graph $G^{\prime}$ which is a forest. We can consider these trees in $G^{\prime}$ respectively. Since $\operatorname{Net}(B, C)$ is a simple net, there are not two vertices from $\operatorname{Net}(B, C)$ which connect a same vertex in $V^{G}-V^{N e t(B, C)}$. Each end vertex corresponds to only one edge whose variable is solved in simple net. Thus, other unsolved variables in $G^{\prime}$ have the same situation as the variables of $G^{\prime}$ in Case 1 . Hence, they can be solved by same procedure as Case 1 .

Case 3: Suppose $m \geq 1$ or $n \geq 1$.

From Case 1 and Case 2, we know that we can solve the variables from all stars or all free simple nets respectively. The rest of the unsolved variables and equations in (3.14) correspond to the graph $G^{\prime}$ which is a forest. We consider the trees of $G^{\prime}$ respectively. By Lemma 3.7, each end vertex of the tree connects only one edge of star or free simple net and any two end vertices are connected by exact one reduced path. Thus, other unsolved variables in $G^{\prime}$ have the same situation as the variables of $G^{\prime}$ in Case 1. We can consider the end vertices in a tree and apply the same procedure as Case 1 or Case 2 to solve all variables in terms of some variables without ambiguity since $G^{\prime}$ is forest.

All variables are presented as

$$
\left(\left(x_{1}: y_{1}\right), \ldots,\left(x_{n}: y_{n}\right)\right)=f\left(\left(x_{1}: y_{1}\right), \ldots,\left(x_{p}: y_{p}\right)\right)
$$

where each component of $f$ is a composition by some maps as (3.9). So, they are homogeneous polynomial of $\left(x_{1}: y_{1}\right), \ldots,\left(x_{p}: y_{p}\right)$.

Let $U:=\left(\mathbf{C P}^{1}\right)^{p}-\left\{\left(\left(x_{1}: y_{1}\right), \ldots,\left(x_{p}: y_{p}\right)\right)\right.$ : for some $1 \leq i \leq p,\left(x_{i}: y_{i}\right)$ is irregular of some equation of (3.14)\}. By Lemma 3.3, $U$ is an open connected set of $\left(\mathbf{C P}^{1}\right)^{p}$. By Lemma 3.4, $f$ defines an embedding from $U \subset$ $\left(\mathbf{C P}^{1}\right)^{p}$ to $\left(\mathbf{C P}^{1}\right)^{n}$. Since $U$ is irreducible, so is $f(U)$ irreducible. Observe that $(0: 1)^{n}=((0: 1), \ldots,(0: 1))$ and $(1: 0)^{n}=((1: 0), \ldots,(1: 0))$ are contained in $f(U)$. We deduce that $(0: 1)^{n}$ and $(1: 0)^{n}$ are in the same irreducible component of $\left\{P_{1}=0, \ldots, P_{c\left(\mathcal{A}_{1}^{*}\right)}=0\right\}$. Recall that irreducible variety minus a subvariety is still a connected set. If $\left(\left(x_{1}: y_{1}\right), \ldots,\left(x_{n}\right.\right.$ : $\left.\left.y_{n}\right)\right)=((1: 0), \ldots,(1: 0))$ (respectively $\left.((0: 1), \ldots,(0: 1))\right)$, then $\mathcal{A}^{*}$ is $\mathcal{A}_{0}^{*}$ (respectively $\mathcal{A}_{1}^{*}$ ). Therefore, condition $(3.12)$ is satisfied at these two points, so there is a curve from $((1: 0), \ldots,(1: 0))$ to $((0: 1), \ldots,(0: 1))$ such that (3.12) is satisfied for any point lying in the curve. This means that we have constructed a one-parameter family of arrangements $\mathcal{A}^{*}(t)$ such that $\mathcal{A}^{*}(0)=\mathcal{A}_{0}^{*}, \mathcal{A}^{*}(1)=\mathcal{A}_{1}^{*}$ and $L(\mathcal{A}(t)) \equiv L\left(\mathcal{A}_{0}\right)$ for all $t \in[0,1]$. Now, we can apply Lattice-Isotopy Theorem and finish the proof of our Main Theorem. 
Rigidity of Differentiable Structure for New Class of Line Arrangements1073

The following corollary is an immediate application of Main Theorem 3.9 .

Corollary 3.10. The homotopy groups of the complement $M\left(\mathcal{A}^{*}\right)$ of a simple arrangement in $\mathbf{C P}^{2}$ depend only on the lattice of $\mathcal{A}$.

From the proof of Main Theorem, we can generalize the result easily.

Definition 3.11. (a) For a sequence of reduced circles $B_{0}, B_{1}, \ldots, B_{n}\left(B_{0}\right.$ can be a vertex), a generalized net of $B_{0}, B_{1}, \ldots, B_{n}$ is a subgraph of $G$, denoted by $N$ et $\left(B_{0}, B_{1}, \ldots, B_{n}\right)$, where

$$
\begin{aligned}
& V^{N e t\left(B_{0}, B_{1}, \ldots, B_{n}\right)}=\bigcup_{i=0}^{n} V^{B_{i}} \bigcup N, \\
& E^{N e t\left(B_{0}, B_{1}, \ldots, B_{n}\right)}=\left\{(v, u) \in E^{G} \mid v, u \in V^{N e t\left(B_{0}, B_{1}, \ldots, B_{n}\right)}\right\},
\end{aligned}
$$

where $N=\left\{v \in V^{G} \mid v\right.$ connects only one vertex in $\bigcup_{i=0}^{n} V^{B_{i}}$ by an edge in $\left.E^{G}\right\}$.

$B_{i}, i=0,1, \ldots, n$ and $N$ are called base- $i$ and node respectively.

The vertex $v$ in $V^{N e t}\left(B_{0}, B_{1}, \ldots, B_{n}\right)$ which connects a vertex of $V^{G}-$ $V^{N e t}\left(B_{0}, B_{1}, \ldots, B_{n}\right)$ by an edge is called end vertex.

(b) If any two non-adjacent vertices of the base-i $B_{i}, i=0, \ldots, n$ are not connected by an edge, any two vertices of two base-i $B_{i}$ and base-j $B_{j}, i, j=$ $0,1, \ldots, n-2$ do not connect a same vertex in base-k $B_{k},(k>i$ and $j)$ by two edges, moreover, any two vertices in the net cannot be connected to a same vertex in $V^{G}-V^{N e t\left(B_{0}, B_{1}, \ldots, B_{n}\right)}$ by two edges, we call $\operatorname{Net}\left(B_{0}, B_{1}, \ldots, B_{n}\right)$ generalized simple net.

(c) The vertex $v$ in $V^{B_{i}}$ is called free if there is no edge connecting $v$ and $V^{B_{j}}(j<i)$. The generalized simple net with free vertex in $V^{B_{i}}$, for all $i=1, \ldots, n$ is called generalized free simple net.

Definition 3.12. An arrangement $\mathcal{A}^{*}$ in $\mathbf{C P}^{2}$ is called generalized simple if the graph $G$ from $\mathcal{A}^{*}$ has the following property: There are finitely many stars and generalized free simple nets, say $\operatorname{St}\left(v_{1}\right), \ldots, S t\left(v_{m}\right)$ and $\operatorname{Net}\left(B_{1_{0}}, B_{1_{1}}, \ldots, B_{1_{k_{1}}}\right), \ldots, N e t\left(B_{n_{0}}, B_{n_{1}}, \ldots, B_{n_{k_{n}}}\right)$, which are pairwise disjoint in $G$ and

$$
\begin{aligned}
& G^{\prime}=G-\left\{( \bigcup _ { i = 1 } ^ { m } ( E ^ { S t ( v _ { i } ) } \cup \{ v _ { i } \} ) ) \cup \left(\bigcup _ { j = 1 } ^ { n } \left(E^{\operatorname{Net}\left(B_{j_{0}}, B_{j_{1}}, \ldots, B_{j_{k_{j}}}\right)}\right.\right.\right. \\
&\left.\left.\left.\cup\left\{v \in V^{\operatorname{Net}\left(B_{j_{0}}, B_{j_{1}}, \ldots, B_{j_{k_{j}}}\right)} \mid v \text { is not an end vertex }\right\}\right)\right)\right\}
\end{aligned}
$$


is a forest.

Clearly, a nice graph is simple and a simple graph is generalized simple.

Now, Main Theorem can be generalized as follows

Theorem 3.13. Let $\mathcal{A}_{0}^{*}$ and $\mathcal{A}_{1}^{*}$ be two generalized simple projective arrangements in $\mathbf{C P}^{2}$. If the lattices of $\mathcal{A}_{0}$ and $\mathcal{A}_{1}$ are isomorphic, then the complements $M\left(\mathcal{A}_{0}^{*}\right)$ and $M\left(\mathcal{A}_{1}^{*}\right)$ are diffeomorphic to each other.

Proof. We notice that the method in Case 2 dealing with the net in the proof of Main Theorem can be used in generalized simple net here. Hence, Theorem 3.13 still holds.

\section{References.}

[1] M. Falk and R. Randell: On the homotopy theory of arrangements II. Adv. Stud. Pure Math., 27 Kinokuniya, Tokyo, 2000.

[2] M. Falk: Arrangements and cohomology. Ann. Comb., 1(2) (1997), $135-157$.

[3] M. Falk: The cohomology and fundamental group of a hyperplane complement. Contemp. Math., 90 (1989), 55-72.

[4] M. Falk: On the algebra associated with a geometric lattice. Adv. Math., 80 (2) (1990), 152-163.

[5] M. Falk: The homotopy types of line arrangements. Invent. Math., 111 (1) (1993), 139-150.

[6] T. Jiang and S. S.-T Yau: Diffeomorphic types of the complements of arrangements of hyperplanes. Compositio Mathematica 92 (1994), $133-155$.

[7] T. Jiang and S. S.-T Yau: Complements of arrangements of hyperplanes. AMS/IP Studies in Adv. Math., 5 (1997), 93-103.

[8] T. Jiang and S. S.-T Yau: Intersection lattices and topological structures of complements of arrangements in $C P^{2}$. Ann. Schola Norm. Sup. Pisa CIA. Sci. (4) XXVI (1998), 357-381.

[9] P. Orlik: Introduction to arrangements. C.B.M.S. Regional Conference Series in Mathematics, No. 72, A.M.S. 
Rigidity of Differentiable Structure for New Class of Line Arrangements1075

[10] P. Orlik and L. Solomon: Unitary reflection groups and cohomology. Invent. Math., 59 (1980), 77-94.

[11] P. Orlik and L. Solomon: Combinations and topology of complements of hyperplanes. Invent. Math. 56 (1980) 167-189.

[12] R. Randall: Lattice-isotopic arrangements are topologically isomorphic. Proc. Amer. Math. Soc. 107 (2)(1989), 555-559.

Department of MSCS, M/C 249

UNIVERSity OF ILLINOIS AT CHICAGO

851 S. Morgan St.,

Chicago, IL 60607-7045

E-mail address: wangs@math.uic.edu

E-mail address: yau@uic.edu

Received OCtoBer 19, 2004. 